\title{
RADIO REFERENCE FRAME STABILITY FROM VLBI DATA
}

\author{
O. J. Sovers and R. N. Treuhaft \\ Jet Propulsion Laboratory, California Institute of Technology \\ 4800 Oak Grove Drive \\ Pasadena, California 91109
}

\begin{abstract}
Intercontinental radio interferometric measurements between NASA's Deep Space Network stations yield a catalog of positions of 106 extragalactic radio sources, uniformly distributed between $-45^{\circ}$ and $+85^{\circ}$ declination. Almost all of the source positions have formal uncertainties between 0.5 and 3 milliarcseconds. Estimates of three independent sets of 32 source coordinates at average epochs 1980.0, 1983.2, and 1984.5 assess the level of stability of the reference frame over a five-year period. Comparisons of the three sets of source coordinates show a number of cases of $>2 \sigma$ differences, particularly betwen the 1984-85 and 1981-83 periods. No sources, however, show $2 \sigma$ shifts among both pairs of epochs. Upper limits of the order of $1 \mathrm{mas} / \mathrm{yr}$ can be placed on the time rates of change of the 32 source coordinates.
\end{abstract}

\section{OBSERVATIONS AND ANALYSIS}

A total of 48 VLBI observing sessions were carried out during 1978-85 on the two DSN intercontinental baselines [California (C) to Spain (S) and to Australia (A)]. The majority were of 24-hr duration, equally divided between CS $(470 \mathrm{hr})$ and CA (415 hr). We employed JPL Mark II data acquisition systems and a bandwidth synthesis technique with channels of $2 \mathrm{MHz}$ bandwidth, spanning about $40 \mathrm{MHz}$ in each radio frequency band. Only dual-frequency measurements were used, to permit elimination of ionospheric delays. Each source was observed a number of times in a given session to cover the full range of mutual visibility. Many different schedules were used on each baseline, but each included observations of $3 \mathrm{C} 273$, the right ascension reference source.

Modeling was performed in Solar System barycentric coordinates defined in terms of the mean equator of J2000.0. We attempted to adhere to the Project MERIT standards (Melbourne et al., 1983) for astronomical constants and Earth models. Tropospheric zenith delays were mapped with the function of Lanyi (1984). The usual value of $12^{h} 29^{m} 6^{s} .6997$ at $\mathrm{J} 2000.0$ was adopted for the right ascension of the reference source 3C 273. A multiparameter diagonally weighted least-squares fit (the "standard fit") determined approximately 1300 parameters from the 6800 delay and delay rate pairs. The fit included session-specific clock and station location parameters, and a troposphere zenith delay at each station for every 3 hour period. The only "global" parameters common to all sessions were the right ascension and declination of each source. A number of variants of the standard fit were also performed, including a search for mismodeling by solving for independent positions of 32 sources in three subsets of the 1978-85 time span. 


\section{SOURCE POSITIONS, COMPARISONS, AND STABILITY TEST}

The standard fit to 1978-85 DSN data produced positional coordinates of 106 sources, which form the JPL 1986-3 catalog. Root-mean-square uncertainties are approximately equal for arc lengths in RA and declination: 2.1 and 2.0 mas, respectively, with the range 0.5 to 3 mas including nearly all sources.

Two recent extragalactic source catalogs [GSFC (Ma, 1986), and IRIS (Robertson et al., 1986)] have 55 (of 101) and 19 (of 26) sources in common with JPL 1986-3, respectively. Since these VLBI programs employ completely different hardware, baselines, observing schedules, and correlation and parameter estimation software, comparison of results was thought to be valuable in identifying residual systematic errors in the VLBI technique. For the common sources the rms formal coordinate uncertainties are substantially smaller than 2 mas for both catalog pairs, indicating that the quality of the three catalogs is essentially comparable. Right ascension rms differences are of the order of 2 mas, declination rms differences are considerably larger ( 6 mas for GSFC-JPL), and the $\chi_{\nu}^{2}$ values (1.2 to 2.3 ) indicate that one or both sets of formal uncertainties are underestimates of the true errors.

The sizable discrepancies between the catalogs led us to perform a number of tests on the DSN data that were designed to uncover systematic problems. One of these was a fit estimating independent positions in each of three time spans for sources with at least 10 intercontinental observations in each period. The 1978-85 DSN observations fall into three time periods : 1978-80 (average epoch 1980.0), 1981-83 (1983.2), and 1984-85 (1984.5). Along with three sets of coordinates of 32 sources, the estimated parameters included a single set of positions for all other sources, and the nutation angles for each intercontinental observing session. Differences of the three sets of source coordinates derived in this manner were calculated, with error estimates taking into account correlations between coordinates at different epochs. While there are four cases of $>2 \sigma$ differences, no source shows $2 \sigma$ shifts between the two pairs of epochs. Of these four sources, only for DA 55 and P $0202+14$ are the directions of the shifts consistent between the two epoch differences. Root-mean-square differences are approximately 2 mas for both pairs of epochs, and $\chi_{\nu}^{2} \mathrm{~s}$ are close to 1 with the exception of that for 1984.5 minus 1983.2 right ascension differences, which becomes 1.03 when one outlier is omitted. From these results, upper limits of the order of 1 mas/yr can be placed on the time variation of the 32 source coordinates. We conclude that the marginally significant positional differences between JPL and other catalogs may arise from unidentified systematic errors.

\section{ACKNOWLDEGMENT}

The research described in this report was carried out by the Jet Propulsion Laboratory, California Institute of Technology, under a contract with NASA.

\section{REFERENCES}

Lanyi, G. E. (1984). Proceedings of the International Symposium on Space Techniques for Geodynamics , Sopron, Hungary, July 9-13, 1984, vol. 2, p. 184.

$\mathrm{Ma}, \mathrm{C}$. (1987). Proceedings of IAU Symposium 128, "The Earth's Rotation and Reference Frames for Geodesy and Geodynamics", 1986, ed. A. K. Babcock, in preparation.

Melbourne, W., Anderle, R., Feissel, M., King, R., McCarthy, D., Smith, D., Tapley, B., and Vicente, R. (1983). USNO Circular No. 167, Washington, D.C.

Robertson, D. S., Fallon, F. W., and Carter, W. E. (1986). Astron. J. 91, 1456. 